\title{
Kondo volume collapse, Kondo breakdown, and Fermi surface transitions in heavy-fermion metals
}

\author{
Andreas Hackl* and Matthias Vojta \\ Institut für Theoretische Physik, Universität zu Köln, Zülpicher Straße 77, 50937 Köln, Germany
}

(Dated: September 14, 2021)

\begin{abstract}
The unconventional critical behavior near magnetic quantum phase transitions in various heavyfermion metals, apparently inconsistent with the standard spin-density-wave scenario, has triggered proposals on the breakdown of the Kondo effect at the critical point. Here we investigate, within one specific scenario, the fate of such a zero-temperature transition upon coupling of the electronic to lattice degrees of freedom. We study a Kondo-Heisenberg model with volume-dependent Kondo coupling - this model displays both Kondo volume collapse and Kondo-breakdown transitions, as well as Lifshitz transitions associated with a change of the Fermi-surface topology. Within a large- $N$ treatment, we find that the Lifshitz transition tends to merge with the Kondo volume collapse and hence becomes first order, whereas the Kondo breakdown transition remains of second order except for very soft lattices. Interesting physics emerges at the zero-temperature endpoint of the Kondo volume collapse: For electrons in two space dimensions, this endpoint is located at the Lifshitz line for a large range of parameters, thus two continuous quantum phase transitions coincide without fine tuning. We analyze the effective Landau theory for such a situation and discuss critical exponents. Finally, we relate our findings to current heavy-fermion experiments.
\end{abstract}

\section{INTRODUCTION}

The past decade has seen growing interest in continuous quantum phase transitions. The associated finitetemperature quantum critical regime is characterized by unconventional dynamics and thermodynamics; in particular, quantum criticality can profoundly modify the metallic properties of both $d$ - and $f$-electron materials. Heavy-electron metals have played a particularly important role in the study of quantum criticality, for these materials lie at the brink of antiferromagnetic (AF) instabilities, and they are readily tuned to an $\mathrm{AF}$ quantum critical point (QCP). The occurrence of this QCP is commonly attributed to a competition between the Kondo screening of the local moments and a tendency to AF ordering due to an inter-moment exchange interaction, as first proposed by Doniach. ${ }^{1}$ Interestingly, various properties near the critical points of materials like $\mathrm{CeCu}_{6-x} \mathrm{Au}_{x}$ and $\mathrm{YbRh}_{2} \mathrm{Si}_{2}$ appear to be inconsistent with the standard spin-density-wave (or Landau-Ginzburg-Wilson) scenario of the magnetic phase transition. ${ }^{2}$ Most prominent here are the singular behavior of the specific-heat coefficient and $E / T$-scaling in inelastic neutron scattering experiments. ${ }^{3}$ These have led to suggestions that the critical point is not simply a magnetic instability of fermionic quasiparticles, but that in addition the Kondo effect, being responsible for the formation of these quasiparticles, breaks down. ${ }^{4-7}$

Assuming that the Kondo breakdown is the primary source of the critical singularities, Senthil et al..$^{6,7}$ have suggested a model where the Kondo breakdown occurs as a topological quantum phase transition between two paramagnetic phases, namely a heavy Fermi liquid (FL) and a so-called fractionalized Fermi liquid $\left(\mathrm{FL}^{*}\right)$, and ordered magnetism is regarded as an epiphenomenon. As in the Doniach picture, the formal control parameter of the transition is the ratio $T_{K} / J_{H}$ between Kondo temperature and Heisenberg exchange of local moments. It is, however, assumed that the local moments form a spin-liquid state without broken symmetries (instead of the standard local-moment antiferromagnetism) in the regime $T_{K} \ll J_{H}$. Such a spin liquid may result from strong quantum fluctuation, induced e.g. by frustrated RKKY or exchange interactions between the local moments. $^{8}$

As phase transitions in heavy-fermion metals are often tuned by external or chemical pressure, the coupling of electronic to lattice degrees of freedom can play a nontrivial role for the overall shape of the phase diagram. This aspect motivates our paper. A well known but spectacular example are the pressure-induced volumecollapse transitions in the trivalent rare earth metals, ${ }^{9}$ like the transition between $\alpha$ and $\gamma$ Ce. In this example, a line of first-order transitions is found in the pressure-temperature plane where the unit cell reduces its volume by about $15 \%$. An order of magnitude change of the Kondo temperature accompanies this transition. These aspects have led to the Kondo volume-collapse model, ${ }^{10,11}$ which explains the transition based on a volume dependence of the Kondo exchange coupling.

In this paper, we shall study a model which contains the ingredients both for the Kondo-breakdown quantum phase transition as well as for Kondo volume-collapse physics, with the goal of obtaining a unified phase diagram of these phenomena. As both transitions can in principle be tuned by pressure, with the inter-atomic distances strongly influencing the Kondo energy scales, an intricate interplay of electronic and lattice degrees of freedom may be expected. Of particular interest will be whether the Kondo-breakdown transition of the type proposed in Ref. 7 survives as a second-order quantum transition once a coupling to the lattice is taken into account. 


\section{A. Summary of results}

Our microscopic analysis is based on a fermionic large$N$ treatment of a Kondo-Heisenberg model in both two and three spatial dimensions, with the Kondo exchange being linearly coupled to static lattice distortions. In the scenario of Ref. 7, the Kondo-breakdown transition between the FL and $\mathrm{U}(1) \mathrm{FL}^{*}$ phases occurs in the presence of two Fermi surface sheets; as a result, a Lifshitz transition generically occurs upon tuning the ratio $T_{K} / J_{H}$ within the heavy Fermi-liquid phase. At such a Lifshitz transition, ${ }^{12}$ well known from weakly interacting Fermi systems, a topological splitting of the Fermi surface occurs, here leading to a transition between Fermi liquids with one and two Fermi sheets (dubbed $\mathrm{FL}_{1}$ and $\mathrm{FL}_{2}$, respectively).

The physical behavior of our system depends on both the ratio $T_{K} / J_{H}$ and the electron-lattice coupling (or the lattice stiffness). Zero-temperature phase diagrams, as function of pressure (which linearly couples to $T_{K}$ ) and $J_{H}$, are shown below in Figs. 2, 3. Interestingly, the above-mentioned Lifshitz transition is easily driven first order upon inclusion of lattice degrees of freedom, and is then accompanied by a jump in lattice volume and Kondo temperature. In contrast, the Kondo breakdown transition $\mathrm{FL}_{2}-\mathrm{FL}^{*}$ remains of second order except for very soft lattices - in the latter case, the Fermi-liquid breakdown occurs from $\mathrm{FL}_{1}$ to $\mathrm{FL}^{*}$ as a strong direct first-order transition. For small $J_{H}$, the behavior is dominated by the interplay of Kondo and lattice physics, and soft lattices admit a separate Kondo volume collapse transition (as in earlier work). This first-order transition merges with the Lifshitz transition at larger $J_{H}$.

For a large range of lattice stiffness values, a zerotemperature endpoint of the Kondo volume collapse occurs in the $p-J_{H}$ phase diagram. Remarkably, in two spatial dimensions this endpoint is quite generically located on the Lifshitz transition line without further fine tuning. This coincidence of two continuous transitions can be further analyzed using an effective theory for coupled Lifshitz and lattice order parameters. It turns out that the electron-lattice coupling leads to non-perturbative effects which are ultimately responsible for the "non-Landau" transition behavior.

\section{B. Relation to earlier work}

Our work merges ideas on the Kondo volume collapse and those of quantum critical behavior near magnetic phase transitions in heavy-fermion compounds. Furthermore, earlier works on quantum Lifshitz transitions play a role as well.

Historically, two main theoretical approaches have been advanced to describe the $\gamma \rightarrow \alpha$ transition of elemental Cerium, namely the Mott transition scenario ${ }^{13}$ and the Kondo volume collapse model. ${ }^{10,11}$ Common to both scenarios is that the $f$ electrons are more localized in the $\gamma$ phase. More recently, the modern dynamical meanfield theory (DMFT) in combination with realistic band structure calculations (LDA+DMFT) was employed, and reasonable agreement with experimental data has been achieved. ${ }^{14}$ The relation between the Kondo volume collapse and quantum criticality was only discussed recently in Ref. 15, where the situation of the endpoint suppressed to $T=0$ was considered. This work did not include competing exchange interactions between local moments, and hence did not contain the physics of magnetic or Kondo criticality.

The breakdown of the lattice Kondo effect at the QCP in heavy fermions was proposed in Refs. 4,5. In particular, the approach of Si et al. ${ }^{5}$ used an extension of DMFT to obtain a scenario of "local quantum criticality", where the onset of magnetism in two dimensions suppresses Kondo screening right at criticality. A somewhat different viewpoint was put forward in Refs. 6,7, where Kondo criticality was discussed in the absence of ordered magnetism. This required the existence of a novel phase, a fractionalized Fermi liquid ( $\left.\mathrm{FL}^{*}\right)$, which is a metallic spin-liquid phase with emergent gauge structure. Central to the Kondo-breakdown transition ${ }^{6,7}$ is that the $f$ electron subsystem becomes insulating, hence the transition can be described as an orbital-selective Mott transition. ${ }^{16,17}$ Ref. 7 studied the mean-field theory to be used below, but the coupling to lattice degrees of freedom was not discussed, and the Lifshitz transition in the FL phase (which will turn out to be important in our analysis) was mentioned, but not investigated in detail. Similar mean-field theories also appear in Refs. 18,19, but not with focus on the Kondo breakdown transition.

Lifshitz transitions in correlated electron systems, with topological changes of the Fermi surface, have been discussed at length in Ref. 20. However, this analysis did not contain the coupling of the electrons to other collective degrees of freedom.

\section{Outline}

The body of this paper is organized as follows: In Sec. II we formulate the Kondo-Heisenberg model supplemented by electron-lattice coupling and discuss its large$N$ solution and the resulting zero-temperature phases. Fully self-consistent numerical calculations of the zerotemperature phase diagrams in the parameter regime of pressure and inter-moment exchange are presented afterwards. Particular attention is paid on the role of dimensionality and geometry of the underlying lattice. We discuss the existence of discontinuous volume collapse transitions that occur in a wide range of our numerically obtained phase diagrams. In Sec. III we focus on the interplay of Lifshitz and volume-collapse transitions in the framework on an effective Landau theory. This analysis is in agreement with the mean-field results for the Kondo lattice, and furthermore shows that, for twodimensional (2d) electrons, the quantum critical endpoint 
of the first-order volume collapse transition tends to coincide with the second-order Lifshitz transition. Fluctuations beyond mean-field theory are discussed in Sec. IV, and a brief summary and outlook concludes the paper.

\section{MICROSCOPIC KONDO-LATTICE THEORY}

The starting point of our analysis is the standard Anderson lattice model, describing conduction electrons on a lattice which hybridize with local atomic $f$-orbitals on the lattice sites.

$$
\begin{aligned}
\mathcal{H} & =\sum_{k \sigma} \epsilon_{k} c_{k \sigma}^{\dagger} c_{k \sigma}+\sum_{i \sigma} \epsilon_{f}^{0} f_{i \sigma}^{\dagger} f_{i \sigma}+U \sum_{i} n_{i \uparrow}^{f} n_{i \downarrow}^{f} \\
& +\frac{1}{\sqrt{\mathcal{N}}} \sum_{k i \sigma}\left(V_{k} e^{-i k R_{i}} c_{k \sigma}^{\dagger} f_{i \sigma}+H . c .\right)
\end{aligned}
$$

The first term describes conduction electrons with band filling $n_{c}$, moving on a two-dimensional (2d) or threedimensional (3d) regular lattice (the former case applies to layered systems with weak electronic inter-layer coupling), $\varepsilon_{f}^{0}(U)$ is the bare $f$ electron energy (Coulomb repulsion), $V_{k}$ the hybridization matrix element, and $\mathcal{N}$ the number of unit cells.

Let us now discuss the influence of volume changes induced by external pressure. ${ }^{21}$ Experimental data on Kondo volume collapse transitions in Ce shows that, throughout the transition, the $f$ level occupation and the $f$ electron levels show only modest changes. ${ }^{22}$ In contrast, the Kondo temperature changes rapidly with volume by orders of magnitude. These results motivate us to neglect any volume dependence of the Anderson lattice model other than that of the hybridization matrix elements. (Note that the Kondo temperature depends exponentially on both the hybridization and the conductionelectron bandwidth. Experimentally, both will be influenced by volume changes. For our qualitative considerations, taking into account the volume dependence of one is sufficient.)

For most of the paper, we will treat the lattice degrees of freedom classically, parameterized by the strain tensor $\hat{\epsilon}$. At a transition between two iso-structural states a suitable order parameter is the trace $\epsilon$ of the strain tensor,

$$
\epsilon \equiv \operatorname{Tr} \hat{\epsilon}=\frac{V-V_{0}}{V_{0}},
$$

where $V_{0}$ is a reference volume. In analogy to Ref. 15 , the hybridization matrix elements are allowed to depend on the local strain $\epsilon_{i}$ by a linearized change of the overlap integral $V_{k}=\langle k|\hat{V}| f\rangle$ between a Bloch electron and a local $f$ electron with its atomic potential $\hat{V}$. Assuming a local hybridization, $V_{k} \equiv V$, we account for the lattice coupling be replacing $\mathcal{H} \rightarrow \mathcal{H}+\mathcal{H}_{c}$ with

$$
\mathcal{H}_{c}=\gamma V \sum_{i \sigma} \epsilon_{i}\left(f_{i \sigma}^{\dagger} c_{i \sigma}+H . c\right)+\frac{B}{2} \frac{V_{0}}{\mathcal{N}} \sum_{i} \epsilon_{i}^{2}
$$

where $\gamma$ is the coefficient of the assumed linear local strain dependence of the hybridization. The last term captures the deformation energy, with $B$ being the bulk modulus. For instance, in a cubic system, $B=\frac{1}{3}\left(c_{11}^{0}+2 c_{12}^{0}\right)$, with bare elastic constants $c_{11}^{0}$ and $c_{12}^{0} \cdot{ }^{21}$

Because of the exponential variation of $T_{K}$ with $J_{K}$, moderate variations of the Kondo coupling $J_{K}$ with volume cause a strong volume dependence of the Kondo temperature. This, in turn, yields a nonlinear equation of state $p(V)=-\left.\frac{\partial F}{\partial V}\right|_{T}=-\left.\frac{1}{V_{0}} \frac{\partial F}{\partial \epsilon}\right|_{T}$. It has been shown ${ }^{10}$ that models of this type yield nonlinear $p-V$ isotherms similar to the van der Waals theory of the liquid-gas transition. The phase diagram of the model from Eq. (1) and (3) has been discussed in Ref. 15, and a zero-temperature volume collapse within the heavy-Fermi liquid phase has been found to occur below a critical value $B^{\star}$ of the bulk modulus.

In this paper, we shall discuss the interplay of volume collapse transitions with a possible breakdown of Kondo screening due to competing inter-moment exchange. To simplify the following approximate treatment, we shall supplement our model by an explicit Heisenberg-type exchange interaction between the local moments, ${ }^{6,7}$ and we shall pass from the Anderson to the Kondo model. ${ }^{23}$ The latter step, justified in the Kondo limit $\frac{V^{2}}{\epsilon_{f}+U}, \frac{V^{2}}{\epsilon_{f}} \rightarrow 0,{ }^{24}$ neglects possible charge fluctuations which we assume to be of minor influence for the structure of phase diagram. Note that we only fix $f$ valence (which is known to not vary drastically across a volume collapse transition) to unity, whereas we still allow for a transition from "itinerant" to "localized" $f$ electrons. ${ }^{7}$ We arrive at a KondoHeisenberg model of the form

$$
\begin{aligned}
\mathcal{H}_{\mathrm{KHM}}= & \sum_{k} \epsilon_{k} c_{k \sigma}^{\dagger} c_{k \sigma}+J_{K}(1+\gamma \epsilon)^{2} \sum_{r} \vec{S}_{r} \cdot \vec{s}_{r} \\
& -\frac{N}{4} J_{K}(1+\gamma \epsilon)^{2}+\frac{B}{2} V_{0} \epsilon^{2} \\
& +J_{H} \sum_{\left\langle r r^{\prime}>\right.} \vec{S}_{r} \cdot \vec{S}_{r^{\prime}}
\end{aligned}
$$

where $\vec{s}_{r}=\frac{1}{2} c_{r \sigma}^{\dagger} \vec{\sigma}_{\sigma \sigma^{\prime}} c_{r \sigma^{\prime}}$ is the local spin density of the conduction electrons. We neglect the influence of lattice distortions on inter-orbital magnetic exchange, ${ }^{21}$ for two reasons: (i) The exponential dependence of Kondo temperature on the strain likely dominates over other instances of electron-lattice couplings. (ii) The physics is primarily influenced by the ratio $T_{K} / J_{H}$, and for qualitative considerations the strain dependence of one of the parameters is sufficient.

In the following, the zero-temperature phase diagram of the model $\mathcal{H}_{\mathrm{KHM}}$ (4) will be determined within a fermionic mean-field approach, ${ }^{7}$ which allows to capture both the volume collapse and the Kondo breakdown transitions. An implicit assumption is that quantum fluctuations of the local moments are large, such that a spin liquid replaces ordered magnetism for $T_{K} \ll J_{H}$. Techni- 
cally, this assumption determines the choice of the meanfield decoupling of $J_{H}$ to be described below.

\section{A. Fermionic large- $N$ theory}

The model (4) cannot be solved analytically as it stands. Instead, much progress can be made by solving it in the large- $N$ approach which has been extensively used in the study of the Kondo lattice and also allows to deal with the magnetic exchange term in Eq. (4). ${ }^{18,19}$

Using a fermionic representation of the local moments allows us to capture phases both with and without Kondo screening. Importantly, for dominant exchange interaction $J_{H}$, one obtains a spin-liquid or valence-bond ground-state, ${ }^{6,7}$ which replaces the metallic antiferromagnet conventionally found in this regime. ${ }^{1}$ Here, we will restrict our attention to phases without broken lattice symmetries, hence tuning the ratio $J_{H} / T_{K}$ drives a transition between a heavy Fermi liquid (FL) and a fractionalized Fermi liquid $\left(\mathrm{FL}^{*}\right)$ - in the latter phase the conduction-electron and local-moment subsystems are decoupled, and the $f$ electrons form a Mott-insulating spin liquid state. The quantum phase transition between FL and $\mathrm{FL}^{*}$ is accompanied by a jump in the Fermi volume. $^{7}$

The large- $N$ approach to the Kondo-Heisenberg model, Eq. (4), extends the local-moment symmetry to the $\mathrm{SU}(N)$ symmetry group. The $N^{2}-1$ components $\left(S_{\sigma \sigma^{\prime}}^{r}\right)_{1 \leq \sigma, \sigma^{\prime} \leq N}$ of a local moment $\vec{S}(r)$ are represented in terms of neutral fermions $f_{r \sigma}$ with a local constraint:

$$
S_{\sigma \sigma^{\prime}}^{r}=f_{r \sigma}^{\dagger} f_{r \sigma^{\prime}}-\frac{\delta_{\sigma \sigma^{\prime}}}{2}, \quad \sum_{\sigma=1}^{N} f_{r \sigma}^{\dagger} f_{r \sigma}=\frac{N}{2}
$$

The interaction terms of Eq. (4) now become quartic. These are decoupled introducing Hubbard-Stratonovich fields $b_{r}(\tau)$ conjugate to $\sum_{\sigma} f_{r \sigma}^{\dagger} c_{r \sigma}$ and $\chi_{i j}(\tau)$ conjugate to $\sum_{\sigma} f_{i \sigma}^{\dagger} f_{j \sigma}$ and the constraint is enforced via Lagrange multipliers $\lambda_{r}(\tau)$. The action corresponding to the representation (5) of Eq. (4) becomes extensive in $N$ by rescaling the coupling constants as $J_{K} \rightarrow \frac{J_{K}}{N}, J_{H} \rightarrow \frac{J_{H}}{N}$. In the $N \rightarrow \infty$ limit the physics is controlled by a saddle point at which the Bose fields condense. We assume a spatially homogeneous mean-field state ${ }^{25,26}$ where $b_{r}$, $\lambda_{r}$, and $\chi_{i j}$ take constant values $b_{0}, \lambda$, and $\chi_{0}$. The mean-field Hamiltonian takes the form ${ }^{27}$

$$
\begin{aligned}
\mathcal{H}_{\mathrm{mf}}= & \sum_{k \sigma}\left(\varepsilon_{k}-\mu_{c}(\epsilon)\right) c_{k \sigma}^{\dagger} c_{k \sigma} \\
& -\sum_{k \sigma}\left(\lambda+2 \chi_{0} \sum_{\vec{r}=\mathrm{n} . \mathrm{n} .} e^{i \vec{r} \cdot \vec{k}}\right) f_{k \sigma}^{\dagger} f_{k \sigma} \\
& -b_{0}(1+\gamma \epsilon)^{2} \sum_{k}\left(c_{k \sigma}^{\dagger} f_{k \sigma}+h . c .\right) \\
& -\frac{J_{K}}{4}(1+\gamma \epsilon)^{2}+V_{0} \frac{B}{2} \epsilon^{2}
\end{aligned}
$$

where we have dropped additional constants. The saddle point equations can be written in the following compact form:

$$
\left\{\begin{array}{c}
\frac{b_{0}(1+\gamma \epsilon)^{2}}{J_{K}} \\
\frac{\chi 0}{J_{H}} \\
1 \\
n_{c}
\end{array}\right\}=\sum_{k}\left\{\begin{array}{l}
G_{f c}\left(k, \tau=0^{-}\right) \\
G_{f}\left(k, \tau=0^{-}\right)\left(\varepsilon_{k f}-\lambda\right) \\
G_{f}\left(k, \tau=0^{-}\right) \\
G_{c}\left(k, \tau=0^{-}\right)
\end{array}\right\}
$$

where $\varepsilon_{k f}=\lambda+2 \chi_{0} \sum_{\vec{r}=\text { n.n. }} e^{i \vec{r} \cdot \vec{k}}$ is the dispersion of the $f$-fermions and $G_{c}, G_{f}$ and $G_{f c}$ are the full conductionelectron, $f$-electron and mixed Green functions, respectively, obtained from the Matsubara Green functions:

$$
\begin{aligned}
& G_{c}\left(i \omega_{n}, k\right)=G_{c}^{0}\left(i \omega_{n}+\mu_{c}-b_{0}^{2} G_{f}^{0}\left(i \omega_{n}, k\right), k\right) \\
& G_{f}\left(i \omega_{n}, k\right)=G_{f}^{0}\left(i \omega_{n}-b_{0}^{2} G_{c}^{0}\left(i \omega_{n}, k\right), k\right) \\
& G_{f c}\left(i \omega_{n}, k\right)=\frac{b_{0} G_{f}^{0}\left(i \omega_{n}, k\right) G_{c}^{0}\left(i \omega_{n}, k\right)}{1-b_{0}^{2} G_{f}^{0}\left(i \omega_{n}, k\right) G_{c}^{0}\left(i \omega_{n}, k\right)}
\end{aligned}
$$

Here $G_{c}^{0}\left(i \omega_{n}, k\right)=\left(i \omega_{n}-\varepsilon_{k}\right)^{-1}$ and $G_{f}^{0}\left(i \omega_{n}, k\right)=\left(i \omega_{n}-\right.$ $\left.\varepsilon_{k f}\right)^{-1}$ are the non-interacting conduction and $f$ electron Green functions.

The equilibrium lattice strain $\epsilon$ minimizes the freeenthalpy function

$$
G(\epsilon)=F(\epsilon)+p V_{0} \epsilon
$$

what leads to the additional self-consistency condition

$$
\begin{aligned}
\epsilon & =\left(\frac{B V_{0}}{\gamma^{3}}-\frac{p V_{0}}{\gamma^{2}}\right)\left(\left(\frac{3}{2}-n_{c}\right) J_{K}\right. \\
& \left.+\frac{8 b_{0}^{2}}{J_{K}}+\frac{B V_{0}}{\gamma^{2}}\right)^{-1}-\gamma^{-1}
\end{aligned}
$$

which completes the set of mean-field equations together with Eq. (8).

\section{B. Zero-temperature phases}

In Eq. (6), the effect of the strain $\epsilon$ is just to renormalize chemical potentials and the Kondo coupling $J_{K}$. Thus, there are two qualitatively different zerotemperature phases of our mean-field model which where already derived in Ref. 7. On the one hand, there is the usual Fermi-liquid (FL) phase when $b_{0}, \chi_{0}, \lambda$ are all nonzero. (Note that $b_{0} \neq 0$ implies $\chi_{0} \neq 0$.) One the other hand, the Kondo hybridization may be zero, $b_{0}=0$, but $\chi_{0} \neq 0$ (and $\lambda=0$ ) - this is the $\mathrm{FL}^{*}$ phase. In this mean-field state the conduction electrons are decoupled from the local moments and form a small ${ }^{7}$ Fermi surface. The local-moment system is described as a U(1) spin liquid with a Fermi surface of neutral spinons. We expect that $\chi_{0} \sim J_{H}$. At finite temperatures the phase transition becomes a crossover - this feature is not captured by mean-field theory but requires to consider the coupling to the compact $\mathrm{U}(1)$ gauge field. ${ }^{7}$ (Spin liquid 


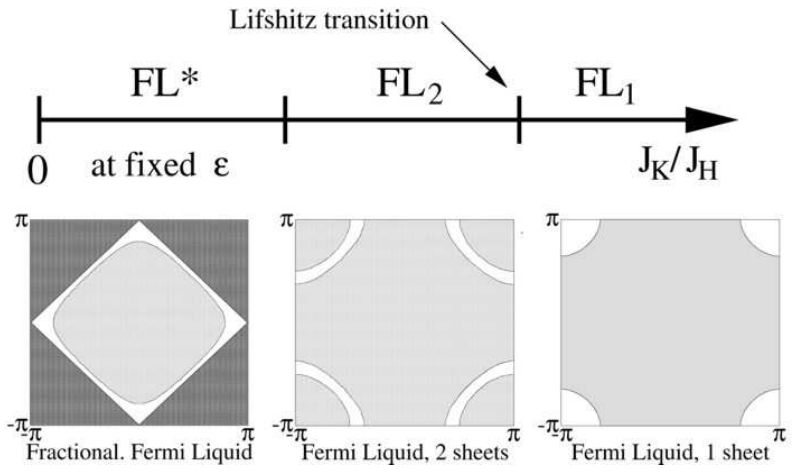

FIG. 1: Fermi surface evolution from FL* to FL, where shaded areas correspond to occupied states. Left: $\mathrm{FL}^{*}$, with one spinon (dark) and one conduction electron (light) sheet. Note that the spinon band is hole-like. Middle: $\mathrm{FL}_{2}$, with two sheets, where the outer one represents heavy quasiparticles with primarily $f$ character. Right: $\mathrm{FL}_{1}$, with one heavyelectron sheet. $\mathrm{FL}_{2}$ and $\mathrm{FL}_{1}$ are separated by a Lifshitz transition where the outer Fermi sheet disappears at critical value of the ratio $J_{H} / J_{K}$. The conduction band has a filling of $n_{c}=0.8$. (The corresponding band structures are also shown in Fig. 4 below.)

states where the gauge group is broken down to $\mathrm{Z}_{2}$ are also possible: Those lead to superconductivity masking the FL-FL* transition $^{6}$ and shall not be considered here.)

We note that the large- $N$ mean-field approach can be expected to be qualitatively correct at zero temperature: The FL phase is stable w.r.t. fluctuations, as is $\mathrm{FL}^{*}$, provided that the $\mathrm{U}(1)$ gauge field is in a deconfined phase. For a further discussion of fluctuation effects we refer the reader to Ref. 7.

The mean-field Hamiltonian can be interpreted as a model of non-interacting quasiparticles with dispersions

$$
E_{k}^{ \pm}=\frac{\varepsilon_{k}+\varepsilon_{k f}}{2} \pm \sqrt{\frac{\left(\varepsilon_{k}-\varepsilon_{k f}\right)^{2}}{2}+4(1+\gamma \epsilon)^{2} b_{0}^{2}}
$$

Now consider the FL phase near the transition (small $\left.b_{0}\right)$. In this case, the two bands $E_{k}^{ \pm}$derive from the $c$ electrons (with weak $f$ character) and the $f$ particles (with weak $c$ character). For small $b_{0}$, both bands together therefore intersect the Fermi energy at least twice so that the Fermi surface consists of two or more sheets (see Fig. 1). Upon increasing $b_{0}$, a topological transition is possible beyond which the Fermi surface intersects the lower band $E_{k}^{-}$only once and the upper band $E_{k}^{+}$is empty (Fig. 4 below). This topological splitting of the Fermi surface is a Lifshitz transition.

Let us point out that the existence of this Lifshitz transition in the FL phase near the Kondo breakdown transition can be seen as a consequence of the fermionic nature of the spinons in the $\mathrm{FL}^{*}$ phase, which then implies the existence of two fermionic bands near the FL-FL* transition (also beyond mean-field). We will comment on other scenarios toward the end of the paper.
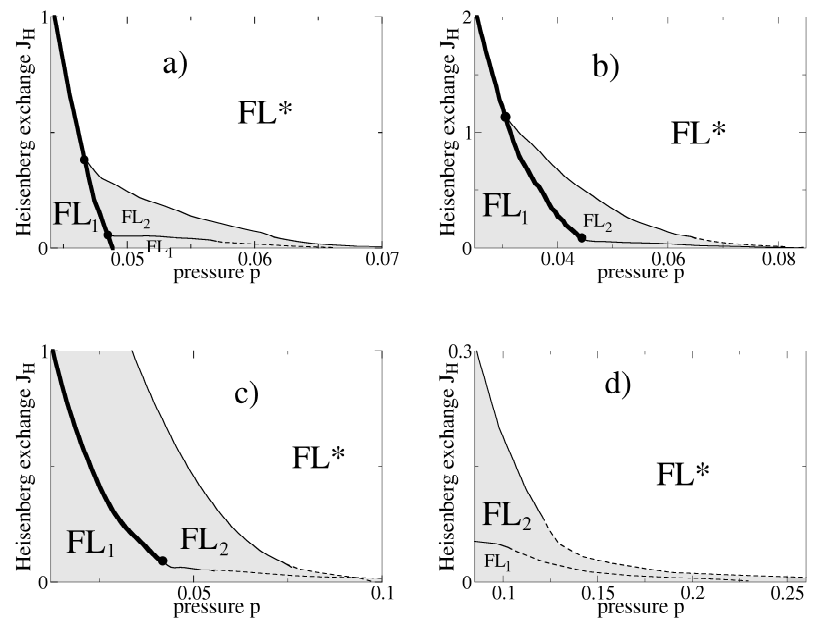

FIG. 2: Large- $N$ ground state phase diagrams of the KondoHeisenberg model (6) on a $2 \mathrm{~d}$ square lattice, as function of pressure $p$ and inter-moment exchange $J_{H}$. The panels a) to d) are for bulk moduli $B=0.005,0.0055,0.007$, and 0.015 ; the other parameters are $t=1, n_{c}=0.8, J_{K}=1.5$ and $\gamma=0.05$. Thin (thick) lines are second (first) order transitions. The Lifshitz transition line separates $\mathrm{FL}_{1}$ and $\mathrm{FL}_{2}$, the Kondo breakdown transition separates FL from $\mathrm{FL}^{*}$, for details see text. (The calculations have been at a low $T=0.005$, the dashed lines are extrapolations of the phase boundaries obtained from runs at lower $T$.)

\section{Numerical results: Phase diagrams}

We have solved the mean-field equations (8) and (10) numerically for different lattice geometries, namely for electrons with nearest-neighbor hopping $t$ on $2 \mathrm{~d}$ square and triangular lattices and for $3 \mathrm{~d}$ cubic lattices. ${ }^{28,29}$ The geometric frustration (here of the triangular lattice) enters our calculation via the dispersion of both $c$ and $f$ particles. Note that conventional magnetic order is excluded from the outset due to the choice of the decoupling scheme. The lattice degrees of freedom are assumed to be $3 \mathrm{~d}$ in all cases, but this does not explicitly enter the mean-field theory (6). The calculations are intended for $T=0$, but are performed at a small finite $T$ for convergence reasons.

We obtain phase diagrams as function of external pressure and inter-moment exchange, with the bulk modulus $B$ and the conduction band filling $n_{c}$ as system parameters $\left(n_{f}=1\right.$ is fixed in the Kondo limit). Most qualitative features are independent of the spatial dimensionality and underlying lattice geometry, therefore we discuss the $2 \mathrm{~d}$ square lattice results unless otherwise noted.

Sample phase diagrams for different values of the bulk modulus are shown in Fig. 2. A sufficiently soft lattice with a correspondingly small bulk modulus makes a first-order transition at the Lifshitz line preferable. Below a critical bulk modulus $B^{\star}$, this first-order lattice transition has no zero-temperature endpoint (Fig. 2a). 


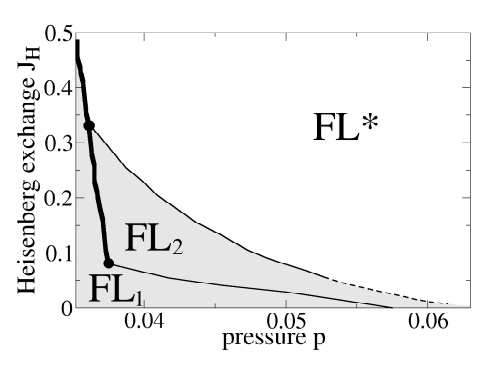

FIG. 3: As in Fig. 2, but for electrons on a 3d cubic lattice, for $B=0.005$.

Instead, it continues to $J_{H}=0$ which is the Kondo volume-collapse transition considered in Ref. 15. By increasing the bulk modulus above $B^{\star}$, the first-order transition shows a quantum critical endpoint which is quite generically located on the Lifshitz line within numerical accuracy (Fig. 2b). Here, for larger values of the intermoment exchange $J_{H}$, the first-order transition coincides with the transition line to the fractionalized Fermi liquid. This type of transition, i.e., a direct first-order transition from a heavy Fermi liquid to a non-Kondo state dominated by inter-moment exchange (which is $\mathrm{FL}^{*}$ here, but will be antiferromagnetic if frustration is reduced) also appears in other theories, ${ }^{30}$ but is usually not observed experimentally. By further increasing the bulk modulus, the first-order volume transition becomes weaker. In particular, the coincidence with the transition to the $\mathrm{FL}^{*}$ phase is shifted to larger values of the parameter $J_{H}$ (Fig. 2c). Beyond a critical value of the bulk modulus, firstorder transitions do no longer exist, Fig. 2d. This behavior turns out to be well described by a Landau theory, to be described in Sec. III below.

As our calculations are performed at small finite $T$, care is required if the characteristic scales of the problem become small. If $J_{H}$ or $T_{K}$ become of order $T$, the transition lines in the phase diagrams start to deviate from the zero-temperature solution. From numerical results at lower temperatures we obtained the asymptotic behavior of the transition lines which is indicated by the dashed lines in Fig. 2.

The large- $N$ results for $3 \mathrm{~d}$ electrons, moving on a cubic lattice, are qualitatively similar to those for $d=2$. An endpoint of the first-order lattice transition line is found (Fig. 3) - whether this endpoint can again be exactly located on the Lifshitz line without fine tuning is difficult to decide, due to limited numerical resolution. (This question shall be investigated using the Landau theory of Sec. III below, with a negative answer.) Above a critical bulk modulus, the first-order volume collapse transition ceases to exist (not shown). Below another critical bulk modulus, the $T=0$ endpoint of the first-order transition disappears. As in $d=2$, the first-order line becomes a direct $\mathrm{FL}_{1}-\mathrm{FL}^{*}$ transition for large values of the intermoment exchange $J_{H}$.

In our numerical results the mean-field bond ampli-
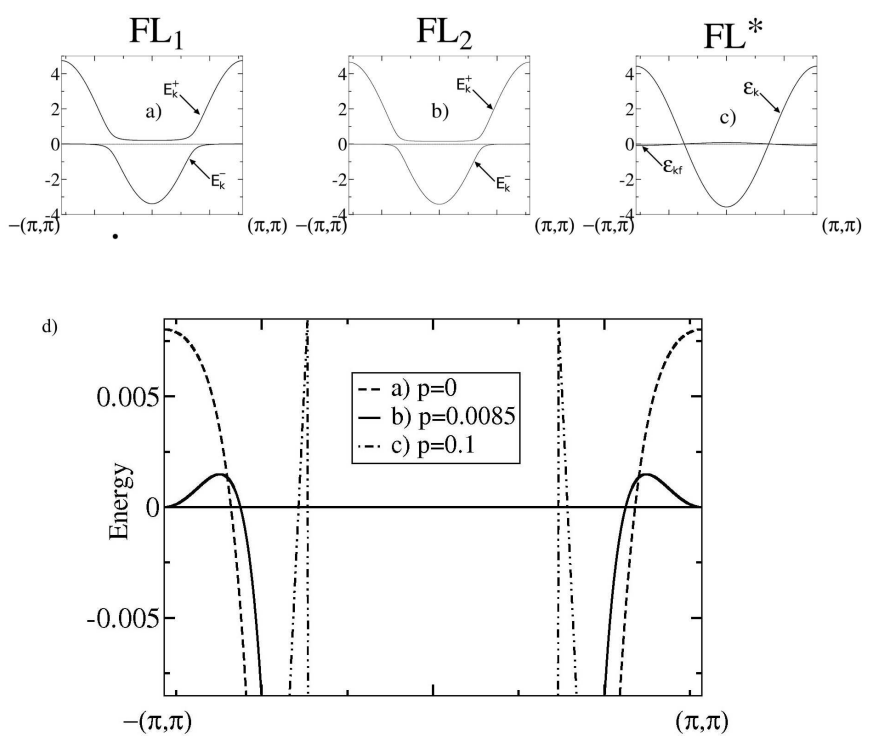

FIG. 4: Quasiparticle dispersions for different parameters along a constant- $J_{H}$ cut of the phase diagram in Fig. 2, for parameters $t=1, n_{c}=0.8, J_{H}=0.1, J_{K}=1.5$, $\gamma=0.05, B=0.01$ and $T=0$. Upper panel: Bands along the $-(\pi, \pi) \rightarrow(\pi, \pi)$ direction for pressures in the a) $\mathrm{FL}_{1}$, b) $\mathrm{FL}_{2}$ (close to the Lifshitz transition), and c) $\mathrm{FL}^{*}$ phases $(p=0,0.0085,0.1$, respectively). Lower panel: Comparison of the low-energy part of the dispersions. Case a) has one heavy electron-like band intersecting the Fermi level. Increasing pressure lowers the energy at the zone boundary and eventually causes the emergence of a hole-like sheet, case b). At higher pressures, the two bands evolve into conduction electron and spinon bands, which each intersect the Fermi energy once - this is the $\mathrm{FL}^{*}$ phase, case c).

tude $\chi_{0}$ is in all aforementioned cases negative, leading to a hole-like spinon dispersion (see also Fig. 4). At a Lifshitz transition, the lower band $E_{k}^{-}$becomes suddenly hole-like, promoting a hole-like pocket which splits from the Fermi surface. Obviously, the emerging holelike pocket will contribute a power-law density of states at the Fermi energy, leading to a singular contribution to the thermodynamic potential - this will be discussed in more detail in the next section.

A comment on the finite-temperature physics is in order: Both the Lifshitz and Kondo breakdown transitions continue to $T>0$ at mean-field level, however, it is clear that both are smeared into crossovers upon inclusion of fluctuation effects. ${ }^{7}$ The first-order lattice transition (for fixed $p, J_{H}$ ) ends in a finite-temperature critical endpoint. These endpoints define a line in a generalized phase diagram which terminates in the $T=0$ endpoint described above.

Let us summarize the physical picture obtained so far. Upon inclusion of lattice degrees of freedom, the Lifshitz transition between $\mathrm{FL}_{1}$ and $\mathrm{FL}_{2}$ tends to become first order (because the system can gain energy by abruptly gapping one of the bands), while the Kondo breakdown transition remains of second order, except for soft lat- 
tices where both transitions merge into a single first-order transition. The calculations further suggest an interesting coincidence of two critical phenomena in the case of $2 \mathrm{~d}$ electrons, namely the endpoint of the volume collapse is located precisely on the Lifshitz transition line. To understand the latter behavior in more detail, we now devise an effective theory for the two phenomena.

\section{LANDAU THEORY FOR LIFSHITZ AND LATTICE VOLUME TRANSITIONS}

Two prominent features of the heavy-fermion model of Sec. II were (i) Lifshitz transitions, where the Fermi surface topology changes with the appearance or disappearance of a Fermi sheet, and (ii) lattice transitions of the volume-collapse type. Note that Lifshitz transitions not only appear in the Kondo-breakdown scenario of Ref. 7, but also in scenarios for metamagnetic behavior inside the heavy Fermi liquid. ${ }^{31}$ These findings motivate to analyze an effective Landau-type theory for Lifshitz plus lattice physics - the mean-field analysis requires less numerical effort than that of the full fermionic large- $N$ theory of Sec. II. The discussion of fluctuation effects will be postponed to Sec. IV.

\section{A. Landau functional}

The vantage point is a conventional Landau theory of an iso-structural volume-changing transition of the crystal lattice - this transition is of liquid-gas type, with a strain order parameter $\epsilon$, Eq. (2). Further, we assume that the system has a Lifshitz transition, where the chemical potential crosses a band edge. Importantly, the lattice parameter $\epsilon$ couples linearly to the tuning parameter of the Lifshitz transition. (Microscopically, $\epsilon$ changes e.g. hopping matrix elements, which in turn moves fermionic bands relative to the chemical potential.)

The band edge crossing the Fermi level gives rise to a non-analytic piece in the Landau effective potential at zero temperature. ${ }^{20}$ The fermion density of states in $d$ dimensions close to the band bottom is:

$$
D(\omega) \propto \Theta(\omega)|\omega|^{\frac{d}{2}-1}
$$

where $\Theta(x)=0$ (1) for $x<0(x>0)$ is the step function. Then, the non-analytic part of the Landau free energy arising from the kinetic energy of the fermions is

$$
F_{\mathrm{F}}(\epsilon)=-\kappa \Theta\left(-\epsilon+\epsilon_{0}\right)\left|\epsilon-\epsilon_{0}\right|^{\frac{d}{2}+1}
$$

where $\kappa>0$ is a coupling constant. For $\epsilon<\epsilon_{0}$ the band becomes occupied, where $\epsilon_{0}$ is the location of the Lifshitz transition.

Choosing a reference volume $V_{0}$ such that $\epsilon_{0}=0$, we can add a regular forth-order expansion in the order pa-
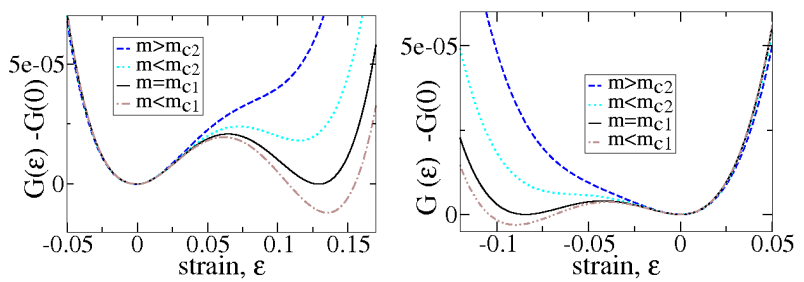

FIG. 5: (Color online) Samples of the mean-field free energy $F(\epsilon)$ (14), for various values of the parameter $v$. The left figure corresponds to $v>0$ and $d=2$, the right figure to $v<0$ and $d=3$. If $m$ drops below $m_{c 2}$, a second minimum evolves at some $\epsilon \neq 0$. If even $m<m_{c 1}$, this minimum becomes the absolute minimum (see also Tables I and II).

rameter to obtain the Landau functional: ${ }^{32}$

$$
\begin{aligned}
F(\epsilon) & =F_{0}-V_{0} p^{\star} \epsilon+\frac{m}{2} \epsilon^{2}-\frac{v}{3} \epsilon^{3}+\frac{u}{4} \epsilon^{4} \\
& -\kappa \Theta(-\epsilon)|\epsilon|^{\frac{d}{2}+1}
\end{aligned}
$$

where $p^{\star}$ is a reference pressure. Microscopically, the mass $m$ is related to the bulk modulus of the material, but will also depend on electronic properties (e.g. the ratio $J_{H} / T_{K}$ in the Kondo lattice case). A cubic term $\epsilon^{3}$ is symmetry-allowed and generically appears in the expansion (14). (Note that in Ref. 15 the reference volume was chosen such that $v=0$ at $T=0$ - this freedom is absent in the present case.)

The physical situation corresponds to pressure tuning and is described by the free-enthalpy functional $G(\epsilon)=$ $F(\epsilon)+p V_{0} \epsilon$, which needs to be minimized to obtain the equilibrium value of the strain $\epsilon$. We shall discuss the arising $T=0$ phase diagram, upon variation of $m$ and $p$, separately for the cases $d=2$ and $d=3$ in the following subsections.

Before proceeding, we remind the reader that the thermodynamic singularities at a Lifshitz transition are in general weak: The ground-state energy follows $F \propto$ $-\Theta(h)|h|^{2}\left(F \propto-\Theta(h)|h|^{5 / 2}\right)$ for electrons in $d=2$ $(d=3)$ as function of the tuning "field" $h$. In contrast, at a usual second-order transition, the free energy scales as $F \propto-|h|^{4 / 3}\left(F \propto-\Theta(h)|h|^{2}\right)$ if $h$ couples linearly (quadratically) to the order parameter, and we have assumed mean-field exponents (and neglected fluctuations). These considerations are relevant, because, for situations with a linear coupling between order parameter and pressure, it is known that lattice effects can render the transition first order due to the compressibility diverging at a finite distance from criticality. ${ }^{33}$ This effect originates from a diverging second derivative $-\partial^{2} F / \partial h^{2}$, and does not occur in the Lifshitz case. In our problem, the compressibility $-\partial^{2} G / \partial p^{2}$ will only diverge at the endpoint of the volume collapse transition. 


\begin{tabular}{c|ccc}
\hline \hline $\boldsymbol{d = 2}$ & $0 \leq|v| \leq v_{c 1}$ & $v_{c 1}<|v| \leq v_{c 2}$ & $v_{c 2}<|v|$ \\
\hline$m_{c 1}$ & $\frac{2}{9} \frac{v^{2}}{u} \Theta(-v)+2 \kappa$ & $\frac{2}{9} \frac{v^{2}}{u} \Theta(-v)+2 \kappa$ & $\frac{2}{9} \frac{v^{2}}{u}+2 \kappa \Theta(-v)$ \\
$m_{c 2}$ & $\frac{1}{3} \frac{v^{2}}{u} \Theta(-v)+2 \kappa$ & $\frac{1}{3} \frac{v^{2}}{u}+2 \kappa \Theta(-v)$ & $\frac{1}{3} \frac{v^{2}}{u}+2 \kappa \Theta(-v)$ \\
\hline \hline
\end{tabular}

TABLE I: Location of critical points from Landau theory in $d=2$, in dependence of the cubic coefficient $v$. The critical values are $v_{c 1}=\sqrt{6 u \kappa}$ and $v_{c 2}=3 \sqrt{u \kappa}$. If $m_{c 1}<m_{c 2}$, a critical endpoint $m_{c 2}$ away from the Lifshitz transition emerges. This is not possible if $0 \leq v \leq v_{c 1}$ since always $m_{c 1}=m_{c 2}=2 \kappa$ then. See text for details.

\section{B. Mean-field phase diagram in $d=2$}

By construction, the Lifshitz transition in Eq. (14) occurs from $\epsilon>0$ to $\epsilon<0$; a lattice transition may occur elsewhere on the $\epsilon$ axis. For $d=2$, the fermionic contribution in Eq. (14) is quadratic in $\epsilon$, i.e., renormalizes the mass $m$ for $\epsilon<0$. In the following, we analyze local minima of the free enthalpy $G(\epsilon)$. We will obtain critical values $m_{c 1,2}$ of the mass $m$ : At $m=m_{c 1}$ the Lifshitz transition (as function of $p$ ) changes from continuous to discontinuous, and at $m=m_{c 2}$ the lattice transition (as function of $p$ ) has its critical endpoint.

A continuous Lifshitz transition is not possible if $G(\epsilon)$ is not minimal at $\epsilon=0$ at any pressure. This is certainly the case if $m-2 \kappa<0$. If $m-2 \kappa \geq 0$, the position of minima depends crucially on sign and magnitude of the cubic coefficient $v$, as depicted in Fig. 5. We distinguish these cases in successive order.

(i) $v>0$. Obviously $G(\epsilon>0)$ can acquire a second minimum if $v$ becomes large such that

$$
\frac{d G(\epsilon)}{d \epsilon}=p-p^{\star}+m \epsilon-v \epsilon^{2}+u \epsilon^{3} \stackrel{!}{=} 0
$$

is possible if $\epsilon>0$. The existence of two minima of $G(\epsilon)$ is a necessary condition for a discontinuous transition to occur. The discriminant of the cubic equation (15) excludes a second minimum if $v^{2} \leq \frac{9}{2} u m$. Obviously the first-order transition line terminates then at the critical endpoint $m_{c 2}=2 \kappa$ as long as $0<v \leq 3 \sqrt{u \kappa}$. In the opposite case, $v>3 \sqrt{u \kappa}$, a second minimum of $G(\epsilon>0)$ develops for pressures that lead to three real solutions of Eq. (15). If $v>\frac{9}{2} \sqrt{u \kappa}$, in this second minimum $G(\epsilon)$ becomes smaller than $F_{0}$ at $p=p^{\star}$ (Fig. 5, left). The Lifshitz transition changes from first to second order at $m_{c 1}=\frac{2}{9} \frac{v^{2}}{u}$. The first-order lattice transition line finally terminates at the critical endpoint $m_{c 2}=\frac{1}{3} \frac{v^{2}}{u}$ where the second minimum vanishes (Fig. 6). Note that $m_{c 1}=m_{c 2}$ if $v \leq v_{c 1}=\sqrt{6 u \kappa}$, which leads to a critical endpoint located at the Lifshitz line (Fig. 6b).

(ii) $v<0$. Our analysis of positive coefficients $v$ does not need to be modified if we repeat all arguments for an order parameter $\epsilon<0$. In this case the Lifshitz transition always becomes continuous at $m_{c 1}=\frac{2}{9} \frac{v^{2}}{u}+2 \kappa$, and the first-order lattice transition line terminates at the critical endpoint $m_{c 2}=\frac{1}{3} \frac{v^{2}}{u}+2 \kappa$. The phase diagram corresponds then always to Fig. 6a.

(iii) $v=0$. In this trivial case the critical endpoint $m_{c 1}=m_{c 2}=2 \kappa$ is situated on the Lifshitz line.

We summarize all different cases in two spatial dimensions in Table I. Qualitatively, all these cases divide into two different types of phase diagrams as depicted in Fig. 6.

If the critical endpoint is on the Lifshitz line, then the effective potential at this special point is $\propto|\epsilon|^{2}\left(\propto|\epsilon|^{3}\right)$ for positive (negative) $\epsilon$. This implies that $\epsilon \propto\left(p-p^{*}\right)^{1 / 2}$ $\left(\epsilon \propto p^{*}-p\right)$ for $p>p^{*}\left(p<p^{*}\right)$ in the vicinity of the endpoint, i.e., for $m=m_{c 1,2}$. The first-order line near this point follows $\left|m-m_{c 1,2}\right| \propto\left|p-p^{*}\right|^{1 / 2}$, and along this line the jump in $\epsilon$ is $\Delta \epsilon \propto\left|m-m_{c 1,2}\right|$. This shows that the critical exponents $\beta$ and $\delta$ do not take their mean-field values, due to the non-analytic behavior of the fermionic part of the Landau potential.

\section{Mean-field phase diagram in $d=3$}

In three dimensions, the singular contribution to the free energy enters with a fractional power which is not contained in the series expansion of the regular part. In addition to the critical parameters $m_{c 1}$ and $m_{c 2}$, it is necessary to introduce further critical parameters $m_{c 1}^{\prime}(u, \kappa, v)$ and $m_{c 2}^{\prime}(u, \kappa, v)$ which mark the onset of local minima of $G(\epsilon)$ at $\epsilon<0$ (Fig. 5b). If pressure is used as control parameter, the relation of $m$ to the critical values $m_{c 1}, m_{c 2}, m_{c 1}^{\prime}$ and $m_{c 2}^{\prime}$ determines the type of phase transition. If $m<0$, generically a discontinuous Lifshitz transition occurs at a critical pressure $p>p^{\star}$. Furthermore, if $v \leq 0$ we obtain $m_{c 1}=m_{c 2}=0$. By varying the parameter $\kappa$ and thereby tuning $m_{c 1}^{\prime}$ and $m_{c 2}^{\prime}$, all entries of Table II can be realized.

From Table II we can read off the phase diagrams possible in $d=3$. Since always $m_{c 1}^{\prime}<m_{c 2}^{\prime}$, the zerotemperature endpoint of the lattice transition is never located on the Lifshitz line (except with further fine-
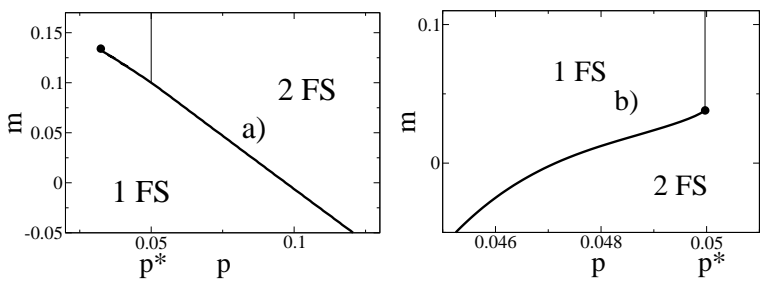

FIG. 6: Two representative mean-field phase diagrams in two dimensions, derived from the Landau theory. Thick/thin lines represent first/second order transitions. If the parameters $m_{c 1}$ and $m_{c 2}$ coincide, the quantum critical endpoint of the lattice transition is located on the Lifshitz transition line (Fig. $6 \mathrm{~b})$. In the other case, $m_{c 1} \neq m_{c 2}$, the first-order lattice transition line turns away from the Lifshitz line and ends at a critical endpoint (Fig. 6a). 


\begin{tabular}{c|ccc}
\hline \hline $\boldsymbol{d}=\mathbf{3}$ & $0 \leq m<m_{c 1}$ & $m_{c 1} \leq m<m_{c 2}$ & $m_{c 2} \leq m$ \\
\hline $0 \leq m<m_{c 1}^{\prime}$ & $1^{\text {st }} \& \mathrm{LS}$ & $1^{\text {st }} \& \mathrm{LS}^{a}$ & $1^{\text {st }} \&_{\mathrm{LS}}$ \\
$m_{c 1}^{\prime} \leq m<m_{c 2}^{\prime}$ & $1^{\text {st }} \& \mathrm{LS}^{a}$ & $1^{\text {st }}$ & $1^{\text {st }}$ \\
$m_{c 2}^{\prime} \leq m$ & $1^{\text {st }} \& \mathrm{LS}$ & $1^{\text {st }}$ & $2^{\text {nd }}$ \\
\hline \hline
\end{tabular}

${ }^{a}$ an additional $1^{\text {st }}$ order transition can occur

${ }^{b}$ two $1^{\text {st }}$ order transitions occur

TABLE II: Type of phase transitions from Landau theory in $d=3$, for $m \geq 0$ and $v>0$. We denote a first-order transition which is also a Lifshitz transition by the entry $1^{\text {st }}$ \& LS. If $m>0$ and $v \leq 0$, we know from the $2 \mathrm{~d}$ case that $m_{c 1}=m_{c 2}=0$ and only the third column can be realized. The latter case leads again to the phase diagram of Fig. $6 \mathrm{~b}$. In the opposite case $v>0$ we always obtain $m_{c 2}>m_{c 1}>0$. From the above table we conclude then that in addition to Fig. 6b, also the phase diagrams of Fig. 7 can be realized.
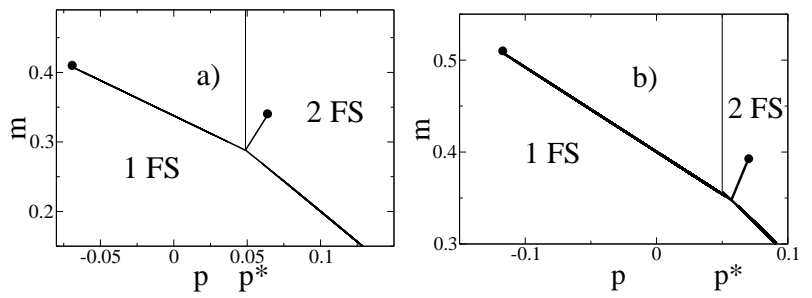

FIG. 7: Additional cases for mean-field phase diagrams that occur in $d=3$. Both situations the first-order lattice transition line bifurcates at a point which a) does [b) does not] coincide with the point where the Lifshitz transition changes from first to second order.

tuning). The behavior along the Lifshitz line can be again of the type shown in Fig. 6a, or there may be additional bifurcations of the first-order lattice transition (Fig. 7).

\section{Conclusions from Landau theory}

It has been shown that, for a range of mass parameters $m$, the Lifshitz transition is rendered discontinuous due to a coupling to the lattice, i.e., a first-order volume collapse coincides with the Lifshitz transition. The volume collapse displays a quantum critical endpoint in the $m-p$ plane. As a specific feature of the $d=2$ theory, the volume-collapse endpoint is located on the Lifshitz transition line in a large parameter regime. (In contrast, in $d=3$ the Lifshitz and volume-collapse transitions separate before the volume-collapse endpoint is reached.)

\section{BEYOND MEAN-FIELD THEORY}

So far, our analysis was restricted to mean-field theory derived either from a large- $N$ or a Landau approach. In this section, we briefly comment on fluctuation effects near the continuous quantum phase transitions of our model system. (It is reasonable to assume that discontin- uous mean-field transitions remain of first order up to a critical temperature where a finite-temperature endpoint of liquid-gas type occurs.)

The second-order Kondo breakdown transition has been investigated in Refs. 7 and 17 - this can lead to interesting non-Fermi liquid behavior, but we shall not repeat the arguments here. The Lifshitz transition is in the universality class of the dilute Fermi gas, with finitetemperature properties discussed e.g. in Ref. 34.

It remains to consider the endpoint of the volumecollapse transition. This has a remarkable feature: The critical endpoint of an iso-structural volume-collapse transition has no critical fluctuations at finite wavevectors. To see this, first note that the strain field $\epsilon$ is related to longitudinal phonons by $\epsilon \sim \nabla_{x}$. $\phi$ where $\phi$ is the displacement field. In continuum elasticity theory, it is well known that, even for a vanishing bulk modulus, a finite shear modulus renders all phonon velocities finite. ${ }^{35}$ A detailed analysis by Cowley ${ }^{36}$ shows that the same is true in crystals: All homogeneous strain fluctuations remain non-critical at the iso-structural volume-collapse transition. (Note that the vanishing of a phonon velocity would imply a structural transition with symmetry breaking.) In other words, at the endpoint under consideration, only the zero-momentum mode becomes critical, and Landau theory is exact for the lattice degrees of freedom. ${ }^{36}$ (Strictly speaking, phonon modes with wavelengths of order sample size become critical.)

For the interesting case of coinciding volume-collapse endpoint and Lifshitz transition, we can briefly discuss the thermodynamics. To this end, temperature corrections to the mean-field parameters $p^{*}$ and $m$ of Eq. (14) have to be taken into account. Due to the underlying Fermi-liquid physics (of the additional bands crossing the Fermi level), these are quadratic in temperature, e.g., $p^{*}=p^{*}(T=0)+\zeta_{1} T^{2}$. Furthermore, the fermionic contribution to Eq. (14) has to be replaced by the free energy of the Fermi gas. Near the Lifshitz transition this can be written as ${ }^{34}$

$$
F_{\mathrm{F}}(\epsilon, T)=-\kappa T^{\frac{d}{2}+1} \Phi(\epsilon / T)
$$

which replaces Eq. (13), and $\Phi(x)$ is a scaling function. Minimizing the free enthalpy $G(\epsilon)$ in $d=2$ now shows that $\epsilon \propto T$ upon raising temperature at the endpoint, i.e., the behavior is dominated by the fermionic part of the free energy. Consequently, the compressibility diverges as $\kappa \propto T^{-1}$, and the specific heat follows $C_{p} \propto T$.

\section{DISCUSSION}

Our study of coupled Kondo-lattice and volumefluctuation physics was motivated by antiferromagnetic quantum critical points in heavy-fermion metals where the proposal of a breakdown of the Kondo effect at criticality has spurred intense interest. Within the scenario of Ref. 7, where a transition between FL and $\mathrm{FL}^{*}$ occurs via the vanishing of the quasiparticle weight on one of the 
two sheets of the FL Fermi surface, we have investigated whether first-order volume transitions may spoil quantum criticality. These calculations merge former investigations on Kondo breakdown and Kondo volume collapse transitions.

Our results show: (i) The Kondo breakdown transition remains intact as a second-order transition (except for very soft lattices), but the Lifshitz transition (i.e. a topological splitting of the Fermi surface) which inevitably appears near the $\mathrm{FL}-\mathrm{FL}^{*}$ transition in the scenario of Ref. 7, tends to become first-order, i.e., occurs concommitantly with a Kondo volume collapse. (ii) Applied to the heavy-fermion metals $\mathrm{CeCu}_{6-x} \mathrm{Au}_{x}$ and $\mathrm{YbRh}_{2} \mathrm{Si}_{2}$, this suggests to experimentally search for (possibly weak) first-order transitions at low temperature near the unconventional candidate critical points. If those are not found, then alternative scenarios of Kondo breakdown should be investigated in more detail ${ }^{4,5}$ - those may not necessarily imply the simultaneous existence of two Fermi sheets near the Kondo breakdown transition. Particularly appealing seems the idea that, at the transition point, quasiparticles on both the large Fermi surface of FL and the small Fermi surface of $\mathrm{FL}^{*}$ (or of the ordered state) become singular, i.e., that two hot Fermi surface sheets coexist, with a "super-large" Fermi surface of volume $1+2 n_{c} \cdot{ }^{37}$ (As a result, the Lifshitz transition is removed from the agenda.) However, a mean-field theory for this scenario is not known. (iii) As an aside, we found that the zero-temperature endpoint coincides with the second-order Lifshitz transition for a large parameter regime in the case of $2 \mathrm{~d}$ electrons. This coincidence of two critical phenomena, i.e. "non-Landau behavior", is rooted in the non-analytic free energy near the topological Lifshitz transition.

\section{Acknowledgments}

We are indebted to M. Garst and A. Rosch for various illuminating discussions, especially on the role of fluctuations at structural phase transitions. Furthermore, we acknowledge helpful conversations with J. Schmalian and T. Senthil. This research was supported by the DFG through the SFB 608 (Köln) and the Research Unit FG 960 "Quantum Phase Transitions".
* ah@thp.uni-koeln.de

1 S. Doniach, Physica B 91, 231 (1977).

${ }^{2}$ H. v. Löhneysen, A. Rosch, M. Vojta, and P. Wölfle, Rev. Mod. Phys. 79, 1015 (2007).

3 A. Schröder, G. Aeppli, R. Coldea, M. Adams, O. Stockert, H. v. Löhneysen, E. Bucher, R. Ramazashvili, and P. Coleman, Nature (London) 407, 351 (2000).

${ }^{4}$ P. Coleman, C. Pépin, Q. Si, and R. Ramazashvili, J. Phys: Condens. Matt. 13, R723 (2001).

${ }^{5}$ Q. Si, S. Rabello, K. Ingersent, and J. L. Smith, Nature (London) 413, 804 (2001); Phys. Rev. B 68, 115103 (2003).

6 T. Senthil, S. Sachdev, and M. Vojta, Phys. Rev. Lett. 69, 216403 (2003).

7 T. Senthil, M. Vojta, and S. Sachdev, Phys. Rev. B 69, 035111 (2004).

${ }^{8}$ For an overview, see e.g.: M. Hermele, T. Senthil, and M. P. A. Fisher, Phys. Rev. B 72, 104404 (2005); F. Alet, A. M. Walczak, and M. P. A. Fisher, Physica A 369, 122 (2006)

9 A. K. McMahan, C. Hushcroft, R. T. Scalettar, and E. L. Pollock, J. of Comp.-Aided Mat. Design 5, 131 (1998).

10 J. W. Allen and R. M. Martin, Phys. Rev. Lett. 49, 1106 (1982).

11 M. Lavagna, C. Lacroix, and M. Cyrot, Phys. Lett. 90A, 210 (1982); J. Phys. F 13, 1007 (1983).

12 I. M. Lifshitz, Sov. Phys. -JETP 11, 1130 (1960) ; J. Exptl. Theoret. Phys. (U.S.S.R) 38, 1569 (1960).

13 B. Johansson, Philos. Mag. 30, 469 (1974).

14 K. Held, A. K. McMahan, and R. T. Scalettar, Phys. Rev. Lett. 87, 276404 (2001); A. K. McMahan, K. Held, and R. T. Scalettar, Phys. Rev. B 67, 075108 (2003).

15 M. Dzero, M. R. Norman, I. Paul, C. Pépin, and J. Schmalian, Phys. Rev. Lett. 97, 185701 (2006).

16 V. Anisimov, I. Nekrasov, D. Kondakov, T. Rice, and M.
Sigrist, Eur. Phys. J. B 25, 191 (2002); S. Biermann, L. de Medici, and A. Georges, Phys. Rev. Lett. 95, 206401 (2005).

17 I. Paul, C. Pépin, and M. R. Norman, Phys. Rev. Lett. 98, 026402 (2007).

18 S. Burdin, D. R. Grempel, and A. Georges, Phys. Rev. B 66, 045111 (2002).

19 B. Coqblin, C. Lacroix, M. A. Gusmao, and J. R. Iglesias, Phys. Rev. B 67, 064417 (2003)

${ }^{20}$ Y. Yamaji, T. Misawa, and M. Imada, J. Phys. Soc. Jpn. 75, 094719 (2006).

21 P. Thalmeier and B. Lüthi, in Handbook of the Physics and Chemistry of the Rare Earths, eds. K. A. Gschneidner and L. Eyring, Vol. 14, Chap. 96, North-Holland, Amsterdam (1991).

22 See e.g. Ref. 10 and references therein.

23 J. R. Schrieffer and P. Wolff, Phys. Rev. 149, 491 (1966).

${ }^{24}$ We consider the particle-hole symmetric case $\epsilon_{f}=-\frac{U}{2}$ here, and neglect possibly arising potential-scattering terms in the Kondo model.

25 Ref. 17 discusses an additional modulated mean-field solution which may occur when the band masses of electrons and spinons have opposite sign. In this phase, $b_{0}$ is not spatially uniform which implies a charge density modulation in the heavy Fermi liquid. To our knowledge, such a phase is not observed experimentally.

26 The fermionic mean-field theory for the local-moment sector can lead to states in which the $\chi$ fields break translational symmetry, resulting in valence-bond solids. For the square lattice, it has been found that ring-exchange interactions stabilize spatially homogeneous mean-field solutions (O. I. Motrunich, private communication). As our interest here is in homogeneous phases, we simply impose translational invariance by hand. 
27 Note the explicit dependence of the Lagrange multipliers $\lambda$ and $\mu_{c}$ on the strain $\epsilon$.

28 The $\mathrm{U}(1) \mathrm{FL}^{*}$ is established as a stable phase in $d=3$ dimensions, ${ }^{7}$ its stability in $d=2$ is a matter of current discussion. ${ }^{29}$ However, even if $\mathrm{FL}^{*}$ is unstable at lowest temperatures, the transition between $\mathrm{FL}$ and $\mathrm{FL}^{*}$ can be relevant for the critical behavior over an intermediate range of energies or temperatures. ${ }^{7,38}$ (On the level of the meanfield calculations, the distinction between $2 \mathrm{~d}$ and $3 \mathrm{~d}$ is only in the dispersion.)

29 M. Hermele, T. Senthil, M. P. A. Fisher, P. A. Lee, N. Nagaosa, and X.-G. Wen, Phys. Rev. B 70, 214437 (2004).

${ }^{30}$ H. Watanabe and M. Ogata, Phys. Rev. Lett. 99, 136401 (2007).

31 S. Viola Kusminskiy, K. S. D. Beach, A. H. Castro Neto, and D. K. Campbell, preprint arXiv:0711.2074.
${ }^{32}$ In principle, the parameters of the Landau expansion, Eq. (14), can be related to those of the microscopic Kondo lattice model of Sec. II, by expanding the mean-field free energy around the numerical mean-field solution at the Lifshitz transition.

33 See e.g. Y. Imry, Phys. Rev. Lett. 33, 1304 (1974).

34 S. Sachdev, Quantum Phase Transitions, Cambridge University Press, Cambridge (1999).

35 L. D. Landau and I. M. Lifshitz, Theory of Elasticity, Theoretical Physics, vol. 7, Butterworth-Heinemann, Oxford (1986).

36 R. A. Cowley, Phys. Rev. B 13, 4877 (1976).

37 T. Senthil, preprint arXiv:cond-mat/0604240.

38 T. Senthil, S. Sachdev, and M. Vojta, Physica B 359-361, 9 (2005). 